\title{
Simulation and theory of fluid-fluid interfaces in binary mixtures of hard spheres and hard rods
}

\author{
Peter G Bolhuis ${ }^{1}$, Joseph M Brader ${ }^{2}$ and Matthias Schmidt ${ }^{3,4}$ \\ ${ }^{1}$ Department of Chemical Engineering, Faculty of Chemistry, University of Amsterdam, \\ Nieuwe Achtergracht 166, 1018 WV Amsterdam, The Netherlands \\ ${ }^{2}$ Institute of Physiology, University of Bern, Bühlplatz 5, 3012 Bern, Switzerland \\ 3 Soft Condensed Matter, Debye Institute, Utrecht University, Princetonplein 5, \\ 3584 CC Utrecht, The Netherlands
}

Received 21 July 2003

Published 20 November 2003

Online at stacks.iop.org/JPhysCM/15/S3421

\begin{abstract}
We consider the free interface between demixed fluid phases in a mixture of hard spheres and vanishingly thin hard rods using Monte Carlo simulations and density functional theory. Both approaches treat the full binary mixture and hence include all rod-induced many-body depletion interactions between spheres. The agreement between theoretical and simulation results for density and orientation order profiles across the interface is remarkable, even for states not far from the critical point. The simulation results confirm the previously predicted preferred vertical (parallel) alignment of rod orientation to the interface plane at the sphere-rich (sphere-poor) side. This ordering should be experimentally observable in phase-separated colloidal rod-sphere mixtures.
\end{abstract}

\section{Introduction}

The gas-liquid interface of a simple substance is characterized by the variation of the density of particles in the direction perpendicular to the interface. The number density is the only order parameter in this situation. Complex systems possess more than a single order parameter and hence may display richer interfacial structure, such as composition fluctuations in mixtures of different components and orientation fluctuations of anisotropic particles. Examples of complex model systems that display fluid-fluid phase separation and hence are suitable to study interfacial structure are mixtures of colloidal spheres and rods.

Much work has been devoted to understanding the bulk phase behaviour of rod-sphere mixtures [1-7]. Among the different techniques employed are computer simulations [1], freevolume $[1,2]$ and liquid integral equation $[4,5]$ theories, as well as experiments with silica spheres mixed with silica coated bohemite rods $[2,3]$ or polymeric rods $[6,7]$. It can be concluded that the gas, fluid and solid phase are thermodynamically stable and that the rod

4 On leave from: Institut für Theoretische Physik II, Heinrich-Heine-Universität Düsseldorf, Universitätsstraße 1, D-40225 Düsseldorf, Germany. 
fugacity plays a role similar to that of inverse temperature in simple fluids. The second control parameter is the ratio of rod length $L$ to sphere diameter $\sigma$; for large enough values, stable bulk fluid-fluid demixing occurs [1].

The simplest model in this context is the binary mixture of hard spheres and vanishingly thin hard rods proposed in [1]. Previous work on this model includes investigation of its phase behaviour with simulation and perturbation theory [1]. Recently, a density functional theory (DFT) [8], built upon Rosenfeld's ideas [9-11], was proposed [12]. Application to the free interface between demixed fluids predicts intriguing ordering behaviour at the interface [13]. By integrating out the rod degrees of freedom, effective pair depletion interactions between spheres and between sphere and a hard wall were derived [14, 15]. For small needle-to-sphere size ratios this was shown to be an accurate description and, using a theory for the effective onecomponent fluid of spheres, purely entropy-induced wetting of the hard wall was found [16]. Rod-induced depletion forces have been experimentally measured and were found to compare well with theoretical predictions [17-19]. Non-vanishing interactions between rods further enrich phase behaviour [20] and correlations between spheres [21].

Despite the existing applications of the rod-sphere DFT [12] to free interfaces [13] and wetting at a hard wall [16], results from the theory have so far been tested against simulation results only in bulk, namely those for the phase diagram and for partial pair distributions between spheres [12]. While theory has passed these (minimal) tests, a full simulation of wetting properties at a hard wall, for example, would be a very demanding task (see e.g. [22] for simulations of a related colloid-polymer mixture). In this work we consider the fluid-fluid interface between demixed fluid phases using Monte Carlo (MC) simulations to obtain density and orientation order parameter profiles which we compare to theoretical results. We find that the theory very accurately describes the simulation results for interface profiles. In particular the simulations confirm that on the needle-rich (sphere-rich) side of the interface the needles are aligned preferentially parallel (vertical) to the interface plane. This effect is present even for vanishing rod-rod interactions and hence is similar, but of different origin, to molecular ordering at the isotropic-nematic interface of hard rods [23].

The paper is organized as follows. In section 2 the theoretical model is defined and brief outlines of both simulation and theoretical methods are given. Section 3 presents results for the interfacial structure. We conclude in section 4.

\section{Model and methods}

\subsection{Definition of the model}

We consider a binary mixture of colloidal spheres with diameter $\sigma$ (species $\mathrm{S}$ ) and needle-like rods with length $L$ and vanishing thickness (species $\mathrm{N}$ ). The pair interaction between colloids as a function of centre-to-centre distance $r$ is that of hard spheres: $V_{\mathrm{SS}}(r)=\infty$ if $r<\sigma$ and zero otherwise. The pair interaction between a sphere and a needle depends on the distance vector from sphere centre to rod centre, $\mathbf{r}$, and on $\operatorname{rod}$ orientation $\Omega$ and is that of hard bodies: $V_{\mathrm{SN}}(\mathbf{r}, \Omega)=\infty$ if both particles overlap and zero otherwise. Due to their vanishing thickness the rods can be regarded as non-interacting: $V_{\mathrm{NN}}=0$ for all distances and orientations. In figure 1 an illustration of the model is displayed.

As thermodynamic parameters we use the sphere packing fraction $\eta=\pi \sigma^{3} \rho_{\mathrm{S}} / 6$ and a reduced needle density $\rho_{\mathrm{N}} \sigma^{3}$, where the number densities are defined as $\rho_{i}=M_{i} / V$, the numbers of particles are $M_{i}$ and the system volume is $V$. The size ratio $L / \sigma$ is a geometric control parameter. We restrict ourselves in the following to $L / \sigma=3$, which is small enough to keep the size of the simulation box reasonably small, and large enough to keep the number of rods necessary to induce phase separation manageable. 


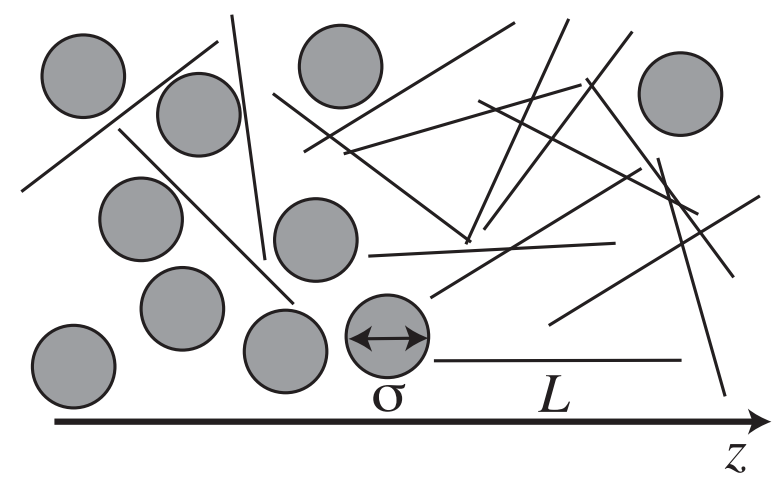

Figure 1. Illustration of the model of hard spheres of diameter $\sigma$ (gray circles) and vanishingly thin needles of length $L$ (lines). The $z$-coordinate is perpendicular to the free interface between sphere-rich (left) and sphere-poor (right) phases.

In the following we will seek to obtain the one-body density distributions of particles across planar fluid-fluid interfaces. For spheres, the dependence is only on the position coordinate, so we have $\rho_{\mathrm{S}}(z)$. For needles, we have $\rho_{\mathrm{N}}(z, \theta)$, where $\theta$ is the angle between rod orientation $\Omega$ and the $z$-direction normal to the interface. For simplification, we consider an angle-averaged needle density given by $\bar{\rho}_{\mathrm{N}}(z)=\int \mathrm{d} \Omega /(4 \pi) \rho_{\mathrm{N}}(z, \theta)$; this can be viewed as the density distribution of rod centres. Needle orientations are analysed by means of an orientation order parameter profile, defined as

$$
P_{2}(z)=\bar{\rho}_{\mathrm{N}}(z)^{-1} \int \frac{\mathrm{d} \Omega}{4 \pi} \rho_{\mathrm{N}}(z, \theta)\left[3 \cos ^{2}(\theta)-1\right] / 2,
$$

where the integrand is $\rho_{\mathrm{N}}$ weighted with the second Legendre polynomial in $\cos \theta$. This is the obvious local generalization with respect to the $z$-direction of the familiar bulk nematic order parameter.

\subsection{Computer simulations}

We perform canonical ensemble MC simulations using a simulation box with periodic boundary conditions, quadratic area in the $x y$-plane, and extended (compared to $x$ and $y$ ) length in the $z$-direction in order to stabilize the interface. The movement of the whole system in the $z$ direction is suppressed by fixing the $z$-coordinate of the origin in the centre of mass of the spheres. The needle fugacity is obtained using Widom's particle insertion method [24].

Detailed parameters in the simulations are as follows. Statepoint A possesses particle numbers $M_{\mathrm{S}}=150$ and $M_{\mathrm{N}}=913$, the box length in the $z$-direction is $12 \sigma$, and the lateral box area is $32 \sigma^{2}$. The resulting densities are $\eta=0.2045$ and $\rho_{\mathrm{N}} \sigma^{3}=2.3776^{\text {Note } 5}$. The sequence of statepoints B is for $M_{\mathrm{S}}=200$ and $M_{\mathrm{N}}=1000,1200,1400$ and 1600 , box length $24 \sigma$ and box area $32 \sigma^{2}$. The corresponding densities are $\rho_{\mathrm{N}} \sigma^{3}=1.302,1.563,1.823$ and 2.083, and $\eta=0.1363$. An investigation of the effect of (lateral) finite size effects was done for the statepoint $\rho_{\mathrm{N}} \sigma^{3}=1.823, \eta=0.1363$, box length $12 \sigma$ and two different (lateral) system sizes. The small system has $M_{\mathrm{S}}=200, M_{\mathrm{N}}=700$ and lateral box area $32 \sigma^{2}$. The large system has $M_{\mathrm{S}}=800, M_{\mathrm{N}}=2800$ and lateral box area $128 \sigma^{2}$.

5 In this work we consider only one fixed size ratio; when comparing results for different $L / \sigma$ it is useful to scale the needle density as $\rho_{\mathrm{N}} L^{2} \sigma[12]$. 
In all simulations the system was equilibrated for $5 \times 10^{4} \mathrm{MC}$ cycles. In each cycle, every particle is moved and/or rotated once, on average. For production, we performed $4 \times 10^{5}$ cycles for each statepoint (except for the case of the largest system where $1 \times 10^{5}$ cycles were done).

\subsection{Density functional theory}

In our theoretical approach the grand potential is expressed as a functional of the one-body density distributions of spheres and needles, $\Omega\left[\rho_{\mathrm{S}}, \rho_{\mathrm{N}}\right]$, using the approximation of $[12,13]$. The advantage over direct application of Rosenfeld's original approach $[10,11]$ for anisotropic particles is that the Mayer functions are correctly recovered in a low-density expansion of the free energy functional. The minimization conditions, $\delta \Omega / \delta \rho_{\mathrm{S}}(z)=0$ and $\delta \Omega / \delta \rho_{\mathrm{N}}(z, \theta)=0$, are solved numerically with a standard iteration procedure. The angular dependence is treated by discretizing $\theta$; our implementation follows closely that of [13], where many more technical details that also apply to the present work can be found.

In order to compare with the simulation results, we impose the same periodicity in the $z$-direction as given by the periodic simulation box. For each statepoint considered during the iteration, the total densities of both components are fixed to the corresponding values from the simulation.

\section{Results}

We plot in figure 2 the fluid-fluid demixing phase diagram as a function of total sphere packing fraction $\eta$ and needle fugacity $\exp \left(\beta \mu_{\mathrm{N}}\right)$, where $\beta$ is the inverse temperature and $\mu_{\mathrm{N}}$ is the needle chemical potential. For high enough values of $\exp \left(\beta \mu_{\mathrm{N}}\right)$ phase separation into a dilute phase (sphere gas) and a dense phase (sphere liquid) occurs. As is known [12], the binodal obtained from DFT is equal to that from perturbation theory [1], and rather accurately describes the simulation results [1]. We also mark the deeply demixed statepoint A and the sequence of statepoints B at constant $\eta$, roughly the value at the critical point, and decreasing $\exp \left(\beta \mu_{\mathrm{N}}\right)$.

As an illustration, in figure 3 we show a snapshot from the simulation of the deeply demixed state. This demonstrates that the fluid-fluid interface can indeed be stabilized in our simulation setup. Obviously, on this microscopic scale, the interface is not sharp, and we next turn to its detailed structure and consider the deeply demixed state at high $\exp \left(\beta \mu_{\mathrm{N}}\right)$ (marked as statepoint $\mathrm{A}$ in figure 2).

As can be expected from the coexistence values of $\eta$ for statepoint $A$, the sphere gas possesses nearly zero density, whereas all sphere particles reside in the liquid phase (see figure 4 for the density profile $\rho_{\mathrm{S}}(z)$ ). A smooth crossover between both plateau values occurs in the interfacial region. The width of the interface is somewhat larger than one might expect for such a deeply demixed state, however, we attribute this to the relatively long needles considered, $L / \sigma=3$. The (orientation-averaged) needle density distribution $\bar{\rho}_{\mathrm{N}}(z)$ shows similar behaviour to $\rho_{\mathrm{S}}(z)$ but is 'out-of-phase', as the dense sphere phase is dilute in needles and vice versa. Finally, the orientation order of the needles, as measured by $P_{2}(z)$, shows that indeed the needles align preferentially parallel to the interface plane on the needle-rich side $\left(P_{2}(z)<0\right)$, and align preferentially perpendicular to the interface plane on the sphere-rich side $\left(P_{2}(z)>0\right)$. Quantitatively, the effect is not very big; recall that the extreme values for perfectly aligned rods are $P_{2}=-1 / 2,1$. The overall agreement between simulation and theoretical results is very remarkable.

This gives us confidence to turn next to the sequence of statepoints approaching the critical point (marked as statepoint B in the phase diagram, figure 2). In this case, fluctuations are expected to be more important. In figure 5, results for $\rho_{i}(z), i=\mathrm{S}, \mathrm{N}$ and $P_{2}(z)$ are shown. 


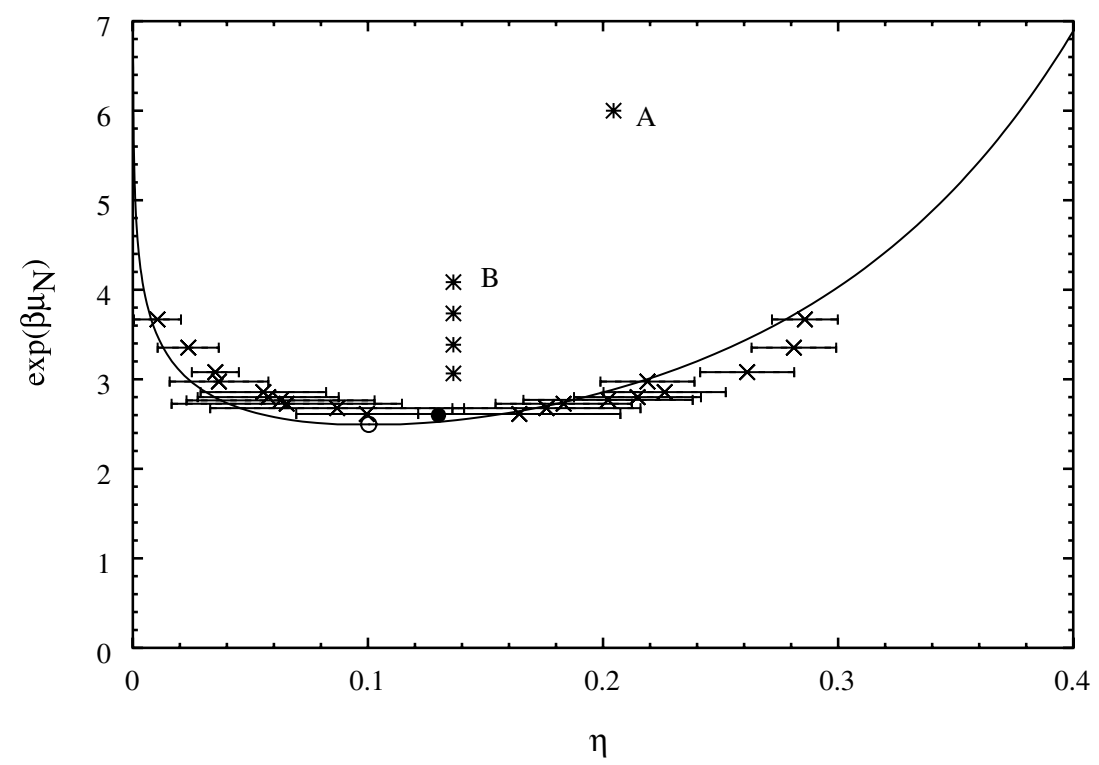

Figure 2. Phase diagram for sphere-needle size ratio $L / \sigma=3$ as a function of sphere packing fraction $\eta$ and needle fugacity $\exp \left(\beta \mu_{\mathrm{N}}\right)$. The results for the binodal and critical point from DFT (line, open circle) and Gibbs ensemble MC simulations [1] (crosses, full circle) are shown. Coexistence is along horizontal tie lines (not shown). Statepoints (stars) where interface structure will be considered are indicated for deeply demixed conditions (A) and a sequence (B) approaching (approximately) the critical point.

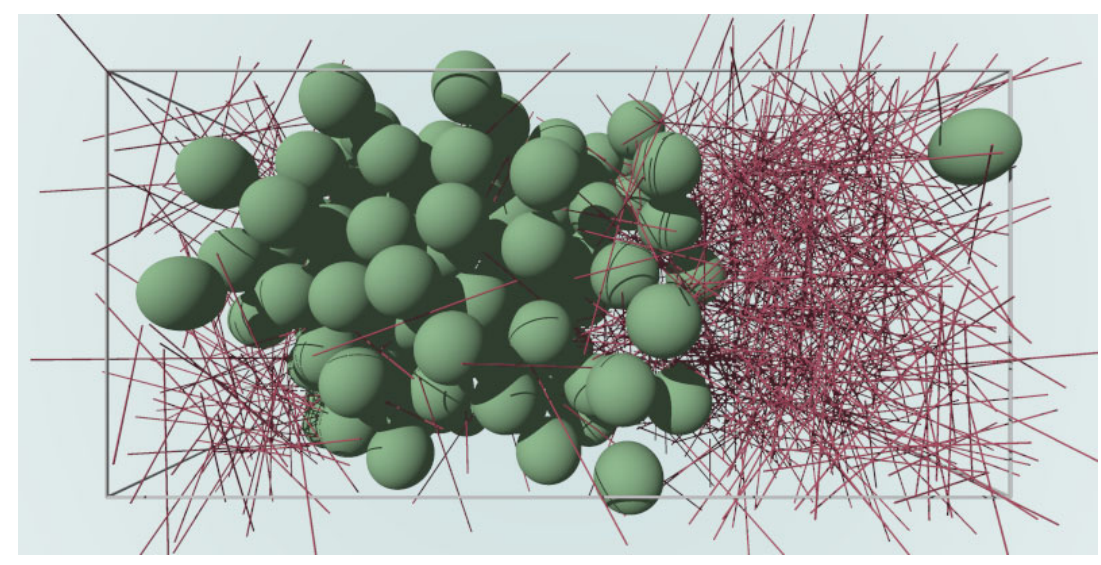

Figure 3. Snapshot from simulation of a phase-separated sphere-needle mixture for size ratio $L / \sigma=3$ and statepoint A (marked in the phase diagram, figure 2). The simulation box is periodic in all three spatial directions.

(This figure is in colour only in the electronic version)

The qualitative behaviour is the same as for the deeply demixed state, however, the variations with $z$ become much smoother as the critical point is approached and the amplitude of $P_{2}(z)$ becomes significantly smaller. The agreement between simulation and theoretical results stays remarkable. Except for a slight shift in $z$ for $P_{2}(z)$ the theoretical curves appear almost as a fit to the MC data. 


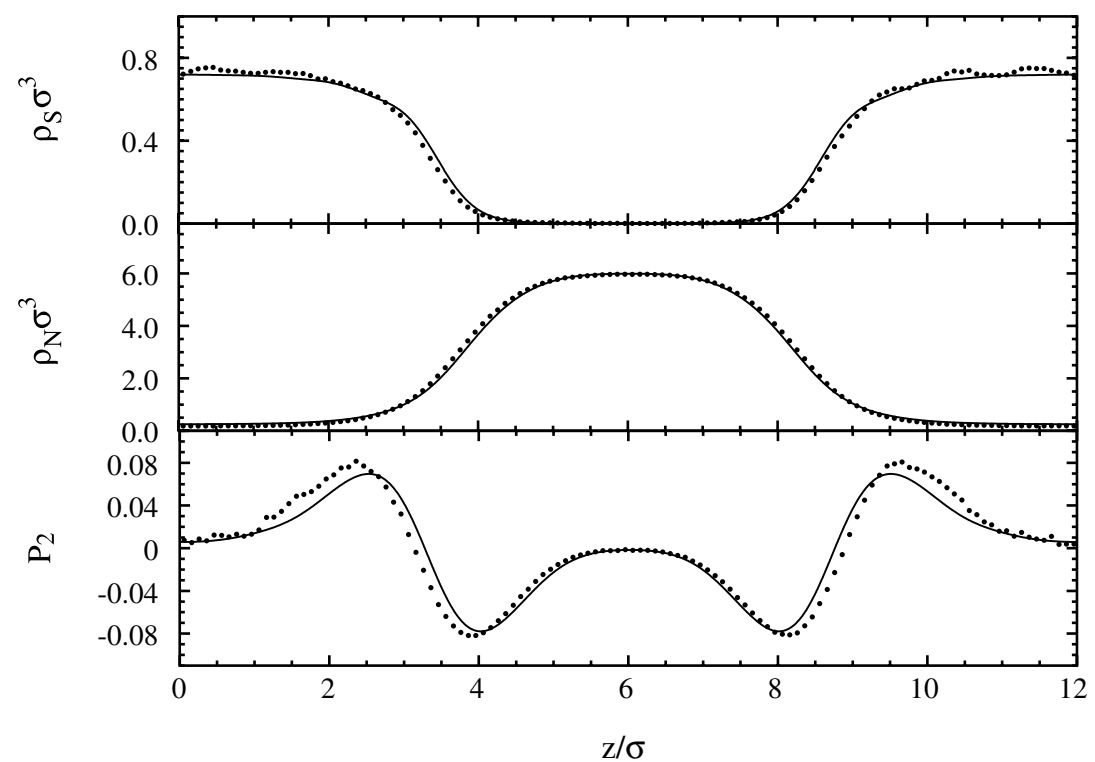

Figure 4. One-body distribution functions of the sphere-needle mixture as a function of the scaled coordinate $z / \sigma$ normal to the free interface between deeply demixed fluid phases. The system is periodic in the $z$-direction with length $12 \sigma$; the statepoint is in the deeply demixed regime, with $\eta=0.2043$ and $\rho_{\mathrm{N}} \sigma^{3}=2.3776$ (see statepoint $\mathrm{A}$ in figure 2). The size ratio is $L / \sigma=3$. The results shown are from simulation (symbols) and theory (curves) for the sphere density profile $\rho_{\mathrm{S}}(z) \sigma^{3}$ (upper panel), orientation-averaged needle density profiles $\rho_{\mathrm{N}}(z) \sigma^{3}$ (middle panel) and orientation order parameter profile $P_{2}(z)$ (lower panel).

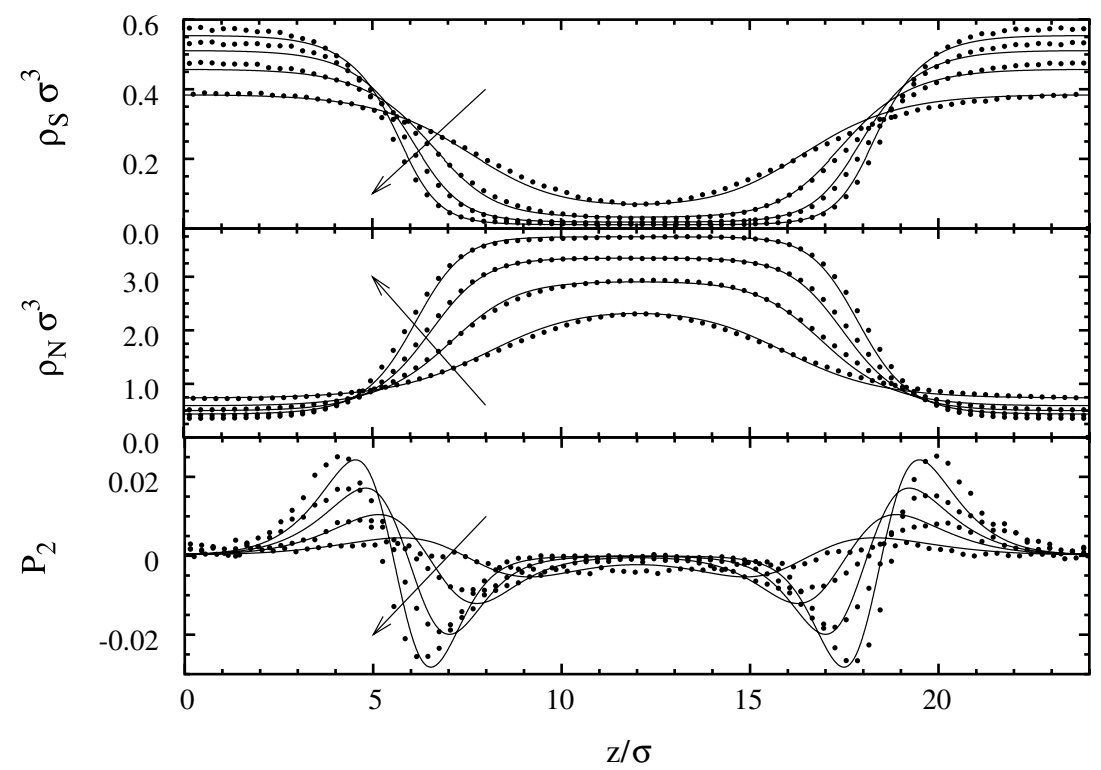

Figure 5. As figure 4, but for a sequence of statepoints with constant $\eta=0.1363$ and increasing (in the direction of the arrow) needle density $\rho_{\mathrm{N}} \sigma^{3}=1.3021,1.5625,1.8229$ and 2.083 (corresponding to the sequence of statepoints B in figure 2). 


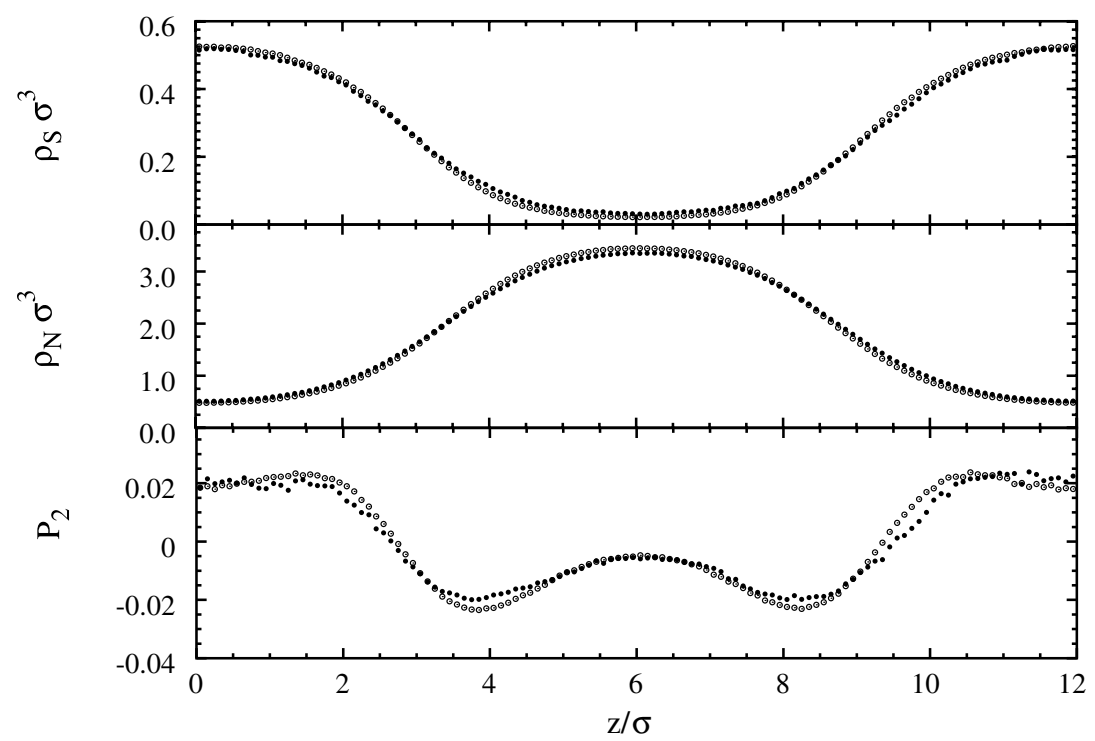

Figure 6. As figure 4 but for system length $12 \sigma, \eta=0.1363$ and $\rho_{\mathrm{N}} \sigma^{3}=1.8229$. Simulation results are shown for different lateral (perpendicular to the $z$-direction) system sizes: a small system with $M_{\mathrm{S}}=200, M_{\mathrm{N}}=700$ and lateral box area $32 \sigma^{2}$ (open symbols), and a large system with $M_{\mathrm{S}}=800, M_{\mathrm{N}}=2800$ and lateral box area $128 \sigma^{2}$ (filled symbols).

For completeness, we give the theoretical results for the surface tension $\gamma$. For the three high rod fugacities within sequence $\mathrm{B}$, these are $\beta \gamma \sigma^{2}=0.500 \pm 0.003,0.343 \pm 0.003$ and $0.202 \pm 0.006$, where the errors given are a conservative estimate and arise from the fact that the bulk values are not perfectly satisfied in the periodic geometry. For this reason no reliable value for $\gamma$ at the statepoint closest to the critical point could be obtained.

As a final investigation, we compare simulation results for the same statepoint, but two different lateral (perpendicular to the $z$-direction) system sizes, differing by a factor of two in linear size (see figure 6). As expected, the larger system possesses slightly smoother profiles. However, the effect is small, and hence we trust that the simulation results do represent the 'bare' density profiles. We have not attempted a complete investigation of the influence of capillary waves, which would require much larger system sizes.

\section{Conclusions}

In conclusion, we have performed a detailed comparison between simulation and theoretical results for the free planar interface between demixed fluid states in a binary mixture of hard spheres and hard rods. The simulation results confirm the previously found ordering of the rods at the interface. While the alignment parallel to the interface on the needle-rich side can be understood in terms of packing against the sphere-rich phase, similar to packing of rods against a hard wall, the normal alignment inside the sphere-rich phase is more subtle. Consider a rod with fixed centre on the sphere-rich side close to the interface. Rotating this rod towards the surface normal will expose less of its shape to the spheres, and hence minimize collisions with spheres. This generates an entropic torque [25] that tends to align rods along the interface normal.

Very good quantitative agreement between results from simulation and theory for the density profiles of both species, as well as the orientation order parameter profile, 
have been found. We have checked that the lateral system dimensions do not play a prominent role but have not attempted to do a systematic investigation of the influence of capillary waves on the interface structure.

We hope to stimulate experimental work on interfaces in rod-sphere mixtures. In particular, measuring the orientation order of rods either in real space or via the induced optical anisotropy would be a very interesting goal.

\section{Acknowledgments}

The work of MS is part of the research programme of the Stichting voor Fundamenteel Onderzoek der Materie (FOM), that is financially supported by the Nederlandse Organisatie voor Wetenschappelijk Onderzoek (NWO).

\section{References}

[1] Bolhuis P and Frenkel D 1994 J. Chem. Phys. 1019869

[2] Vliegenthart G A and Lekkerkerker H N W 1999 J. Chem. Phys. 1114153

[3] Koenderink G H, Vliegenthart G A, Kluijtmans S G J M, van Blaaderen A, Philipse A P and Lekkerkerker H N W 1999 Langmuir 154693

[4] Chen Y L and Schweizer K S 2002 J. Chem. Phys. 1171351

[5] Chen Y L and Schweizer K S 2002 Langmuir 187354

[6] Vliegenthart G A 1999 Dissertation Utrecht University

[7] Koenderink G H, Aarts D G A L, de Villeneuve V W A, Philipse A P, Tuinier R and Lekkerkerker H N W 2003 Biomacromolecules 4129

[8] Evans R 1992 Fundamentals of Inhomogeneous Fluids ed D Henderson (New York: Dekker) p 85

[9] Rosenfeld Y 1989 Phys. Rev. Lett. 63980

[10] Rosenfeld Y 1994 Phys. Rev. E 50 R3318

[11] Rosenfeld Y 1995 Mol. Phys. 86637

[12] Schmidt M 2001 Phys. Rev. E 63 050201(R)

[13] Brader J M, Esztermann A and Schmidt M 2002 Phys. Rev. E 66031401

[14] Yaman K, Jeppesen C and Marques C M 1998 Europhys. Lett. 42221

[15] Roth R 2003 J. Phys.: Condens. Matter 15 S277

[16] Roth R, Brader J M and Schmidt M 2003 Europhys. Lett. 63549

[17] Lin K, Crocker J C, Zeri A C and Yodh A G 2001 Phys. Rev. Lett. 87088301

[18] Lau A W C, Lin K H and Yodh A G 2002 Phys. Rev. E 66020401

[19] Helden L, Roth R, Koenderink G H, Leiderer P and Bechinger C 2003 Phys. Rev. Lett. 90048301

[20] Adams M, Dogic Z, Keller S L and Fraden S 1998 Nature 393349

[21] van der Schoot P 2002 J. Chem. Phys. 1173537

[22] Dijkstra M and van Roij R 2002 Phys. Rev. Lett. 89208303

[23] Shundyak K and van Roij R 2001 J. Phys.: Condens. Matter 134789

[24] Frenkel D and Smit B 2002 Understanding Molecular Simulations 2nd edn (San Diego, CA: Academic)

[25] Roth R, van Roij R, Andrienko D, Mecke K R and Dietrich S 2002 Phys. Rev. Lett. 89088301 